\title{
Influence of lubricant addition on dynamics of a single drop impacting onto a very hot substrate
}

\author{
Marija Gajevic Joksimovic*, J. Benedikt Schmidt, llia V. Roisman, Jeanette Hussong, \\ Cameron Tropea \\ Institute for Fluid Mechanics and Aerodynamics, \\ Technical University Darmstadt, Darmstadt, Germany \\ *Corresponding author email: gajevic-joksimovic@sla.tu-darmstadt.de
}

\begin{abstract}
In the present experimental study the effect of graphite lubricant additives on the dynamics of a single drop impact onto a very hot surface, accompanied by intensive nucleate or film boiling and thermal atomization, has been investigated. The impact has been observed using a highspeed video system. The observations allow to identify the impact regime and to measure the drop residence time from collision to complete rebound, disintegration or evaporation, depending on the thermodynamic regime of the event. The residence time of the drop is measured as a function of lubricant concentration and of the initial temperature of the heated surface. The measurement results are compared to existing experimental data obtained for pure water drops. Additionally, an existing mathematical model developed for pure water impact was re-calibrated for various lubricant mixtures.
\end{abstract}

\section{Keywords}

Drop Impact, Graphite Lubricant, Heat Transfer, Nucleate Boiling.

\section{Introduction}

Single drop impact onto a hot surface occurs frequently over a broad range of applications in numerous industrial sectors. For instance, spray cooling represents a high performance technology used in applications such as die forging, hot mill rolling, cooling of powerful electronics, etc. An extensive overview of spray cooling technology can be found in [1-6]. In most of these cases a pure liquid - distilled water or other liquids - are used in laboratory studies. However, the fluids used in industrial applications, for example for cooling of dies or mechanical parts in the forging process, are often a multi-component mixture of water and lubricants, which are not only intended for cooling, but also for reducing wear and friction.

Many phenomena accompanying the impact of a pure water drop onto a hot substrate have already been scrutinized in literature [7-11], including thermodynamic boiling phenomena (e.g. single phase cooling, nucleate boiling, transitional boiling and film boiling) and different impact outcomes (e.g. deposition, rebound, breakup). The outcome of a drop impact onto a hot surface differs greatly for different parameters such as drop diameter and impact velocity, substrate temperature as well as the fluid properties and the surface wettability. Outcome descriptions of drop impact onto a hot surfaces can be found for example in $[12,13]$. To characterize such impacts some additional parameters are frequently measured and observed, in particular the residence time of the drop liquid on the substrate [14].

The influence of additional components such as lubricants and/or other additives on the drop impact outcome, heat flux regimes and residence time remains largely unknown. The main objective of the present experimental study is the examination of the influence of graphite based industrial lubricants on the drop residence time and drop impact regimes during the interaction between graphite-water drops with a heated substrate. Special attention was given to the nucleate boiling regime, where comparisons were drawn with an existing residence time model for water. The experiments were performed for different volume concentrations of lubricants and 
surface temperatures and covering all of the above mentioned outcome regimes. Finally, we show that the residence time of the lubricant containing drop is proportional to the corresponding time of the pure liquid, which indicates that generally the dynamics of drop impact is very similar. However, the residence time monotonically increases with the lubricant concentration. This effect can be explained by the influence of the dispersed phase on the effective thermal properties of the liquids and by the formation of a deposited layer of the solid particles of the lubricant, which influences the intensity of heat transfer.

\section{Experimental methods}

\section{Configuration of experimental setup}

The experimental setup is designed to observe and characterize the impact of the single drop onto a hot surface. A schematic representation of the setup is shown in Fig. 1(a).

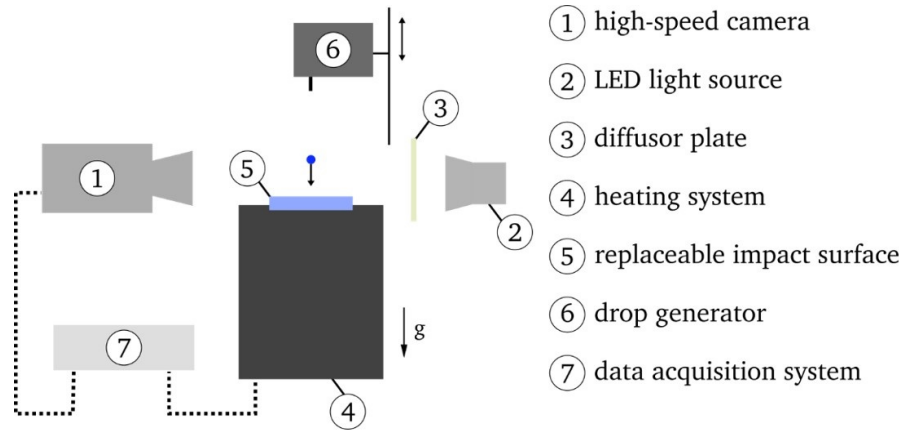

(a) Schematic representation of the experimental installation.

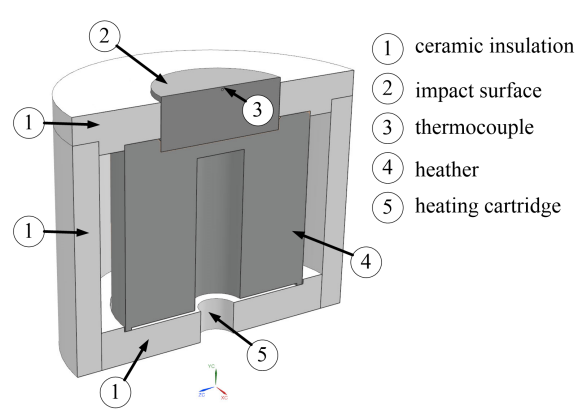

(b) Sectional view of the heating system.

Figure 1. Experimental facility.

The experimental setup presented in Fig. 1(a) can be divided into four major subsystems: an observation system comprising a high-speed camera (1) with LED illumination (2) and diffusor plate (3); a heating system with a temperature controller (4) and a replaceable impact surface (5); the drop generation system (6); and a computer (7) for data acquisition and control of the experimental flow.

Drops are generated with a syringe pump (World Precision Instruments) and a blunt hypodermic needle (Braun Sterican). The desired diameter of the drop can be selected by the displacement of the syringe. The position of the needle can be changed above the impact surface using a linear motor, thus achieving different impact velocities of the falling drops.

A CMOS high-speed camera (Vision Research Phantom V12.1), which can achieve a maximum resolution of $1280 \times 800$ pixel at $6242 \mathrm{fps}$, is used to record side-view images and videos of the drop impact. An image based auto trigger engages when the droplet arrives in the proximity of the target. The high-speed camera is additionally equipped with a $60 \mathrm{~mm}$ macro lens (Nikon AF NIKKOR 1:2.8 D) and spacer rings (Nikon PK).

An LED spotlight (Veritas miniConstellation 120C28) with a power of $120 \mathrm{~W}$ is used for illumination. This illumination is placed behind the drop (and directed co-linear with the high-speed camera) resulting in shadowgraphy imaging. A diffuser plate with a diffusion angle of $30^{\circ}$ is placed between the LED illumination and the impact zone of the drop in order to achieve more uniform illumination of the falling drop.

The heating system consists of a replaceable impact target and a heated aluminium cylinder. The impact target is a stainless steel cylinder with a diameter of $50.8 \mathrm{~mm}$, which is embedded in a heated coaxial aluminium cylinder. The impact surface of the target is mirror polished, with an average roughness of $0.05 \mu \mathrm{m}$. In order to achieve the desired temperature of the impact surface, a $315 \mathrm{~W}$ cartridge heater (hotset hotrod HHP) is mounted within the aluminium heating 
cylinder, on the top of which the impact surface is placed. Additionally, ceramic insulation material (C610) with a very low thermal conductivity is placed around the heated cylinder to provide insulation of the side walls.

The temperature of the stainless steel impact surface is controlled by a PID thermo-controller (HOTSET c448), together with a type-J thermocouple placed $0.5 \mathrm{~mm}$ below the upper surface. A more detailed sectional view of the heating system can be seen in Fig. 1(b). Given the high thermal conductivity of the target material, the temperature difference between the thermocouple and the impact surface can be neglected and the surface temperature can be approximated to be equal to the measured substrate temperature provided by the thermocouple.

\section{Preparation of lubricants}

The working fluid used in experiments is a water based dilution of an industrial lubricant LUBRODAL F105 [15], which is produced by the company Fuchs LUBRITECH. This lubricant can be described as a water miscible graphite dispersion used for die lubrication and different forming operations, from simple to complex. It is initially delivered as a concentrate and has to be diluted with water prior to use. Dilution ratios from 1:2 to 1:20 are common in the die forging industry. The most appropriate dilution ratio depends on the difficulty of forming operation as well as on the part geometry.

In the experimental campaign, dilutions with volume concentration ratios 1:2, 1:4 and 1:8 were initially used for determining residence time. Later, additional experiments with volume concentrations of 1:3,1:5, 1:6 and 1:7 were performed for purposes of determining the influence of lubricant concentration on drop residence time in the nucleate boiling regime.

\section{Mathematical model for residence time in the nucleate boiling regime}

The theoretical basis for modeling drop impact onto a heated solid surface was scrutinized in detail in the doctoral thesis [7] as well as in [14]. In this chapter these existing theoretical models are adapted to the water-lubricant mixture.

The contact residence time $t_{\mathrm{r}}$ is defined as the time span between the initial impact of the drop and the instant of drop rebound (for example, in the film boiling regime) or by the duration of complete evaporation of the deposited drop (in nucleate boiling or transitional boiling regimes). To achieve an estimate of the total evaporation time of the deposited drop in the nucleate boiling regime, a heat balance model from $[16,17]$ is needed. The target is assumed to be a semiinfinite wall, where $x$ is the longitudinal wall coordinate, with its origin at the upper surface of the substrate. The material properties of the solid are described using the temperature diffusivity $\alpha_{w}$. The one-dimensional heat conduction equation in the wall is given as:

$$
\frac{\partial T_{\mathrm{w}}}{\partial t}=\alpha_{\mathrm{w}} \frac{\partial^{2} T_{\mathrm{w}}}{\partial x^{2}} .
$$

Before impact, a uniform temperature field in the target is assumed and will be denoted $T_{\mathrm{w} 0}$. Due to the presence of the heating cartridge, we can also assume that the temperature of the lower wall of the substrate does not change with time, so it remains constant at $T_{\mathrm{w} 0}$. Since the post-impact state of drop induced cooling is assumed to be in the nucleate boiling regime, we also assume that the temperature of the substrate upper surface is equal to the saturation temperature of the fluid $T_{\text {sat }}$.

With boundary conditions provided as such, the analytical solution of the heat conduction equation in the wall is found as [7]:

$$
T_{\mathrm{w}}(x, t)=T_{\mathrm{sat}}+\left(T_{\mathrm{w} 0}-T_{\mathrm{sat}}\right) \operatorname{erf}\left(\frac{x}{2 \sqrt{\alpha_{\mathrm{w}} t}}\right),
$$

where $x$ is the longitudinal coordinate of the semi-infinite wall. 
Using Eq. (2), the specific heat flux on the upper wall surface is then calculated as:

$$
\dot{q}=\left.\lambda_{\mathrm{w}} \frac{\partial T_{\mathrm{sat}}}{\partial x}\right|_{x=0} .
$$

If we express the difference between the wall temperature $T_{\text {wall }}$ and the saturation temperature $T_{\text {sat }}$ as:

$$
\Delta T_{\mathrm{w}}=T_{\text {wall }}-T_{\text {sat }},
$$

the heat flux is then directly expressed combining Eqs. (2) and (3) [7]:

$$
\dot{q}=\frac{e_{\mathrm{w}} \Delta T_{\mathrm{w}}}{\sqrt{\pi t}} .
$$

By equating the heating energy required for complete evaporation with the heat energy which is removed from the substrate material, the total evaporation time can be calculated as [7]:

$$
t_{\mathrm{r}}=\pi\left(\frac{\rho L^{*} d_{0}}{12 k_{\mathrm{w}} e_{\mathrm{w}} \Delta T_{\mathrm{w}}}\right)^{2},
$$

where $L^{*}=L+\Delta H_{0}$ denotes the sum of the latent heat of vaporization $L$ and the enthalpy change between the initial state and the saturated state of the drop liquid, $\Delta H_{0}$. Thermal properties of the substrate material are taken into account through the effusivity $e_{\mathrm{w}}$.

The empirical constant $k_{\mathrm{w}}$ accounts for additional effects that cannot be captured by the model, like surface wettability or the general condition of the surface. It can generally be assumed that $k_{\mathrm{w}}$ depends on the physical properties of the order of $\mathcal{O}(1)$. Following the work of [7], its value is determined as $k_{\mathrm{w}}=1.9$ for pure water.

\section{Results and Discussion}

\section{Observations of impacts of lubricant consisting drops}

In order to obtain a reliable data basis, a broad range of experimental parameters, impact scenarios and heat transfer regimes were tested. The most important parameter which directly determines the impact outcome is the wall overheat $\Delta T_{\mathrm{w}}=T_{\mathrm{w} 0}-T_{\mathrm{sat}}$.

Various regimes of drop impact are shown in Fig. 2. These regimes are similar to the regimes of drop impact of pure liquids. With an increase in wall overheat, the following heat transfer regimes occur: nucleate boiling, transitional boiling, thermal atomisation and finally, filmboiling, once the Leidenfrost point has been exceeded. In order to subject the drops to the full range of possible regime outcomes, the wall overheat is varied in the range: 50 to $320{ }^{\circ} \mathrm{C}$.

Additionally, lubricant-to-water ratios were varied, resulting in several different batches of fluid. Mixture batches in volume concentration ratios 1:2, 1:4 and 1:8 are chosen to cover the wide range of different effects. As will be subsequently demonstrated, the impact outcome of the mixture asymptotically approaches the water impact scenario past the concentration of $1: 8$. A further decrease of lubricant concentration was therefore not performed.

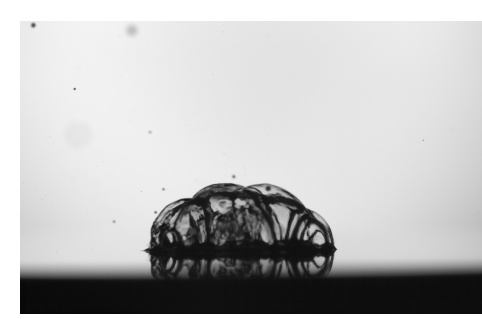

(a) Nucleate boiling.

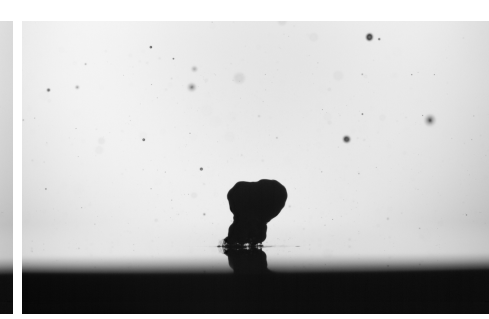

(b) Transitional boiling.

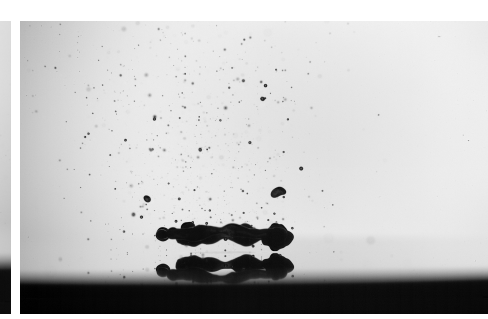

(c) Film boiling.

Figure 2. Different regimes observed during drop impact with impact parameters $d_{0}=2.3 \mathrm{~mm} U_{0}=1.3 \mathrm{~m} / \mathrm{s}$. 


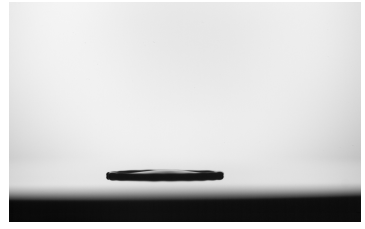

(a) $t=2 \mathrm{~ms}$

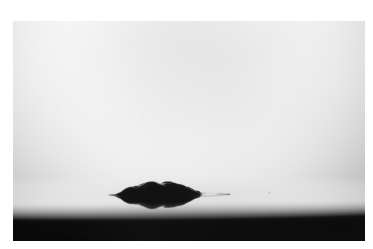

(b) $t=104 \mathrm{~ms}$

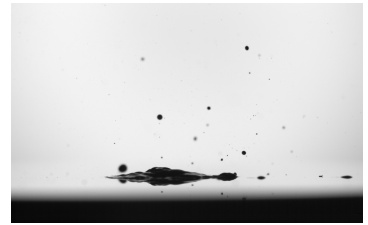

(c) $t=317 \mathrm{~ms}$

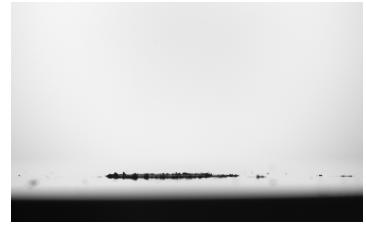

(d) $t=1397 \mathrm{~ms}$

Figure 3. Dilution 1:8 - Different phenomena for different time instants: drop impact with initial substrate temperature $T_{\mathrm{w} 0}=150{ }^{\circ} \mathrm{C}$, drop diameter $d_{0}=2.3 \mathrm{~mm}$ and impact velocity $U_{0}=1.7 \mathrm{~m} / \mathrm{s}$.

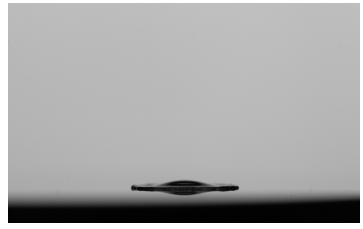

(a) $t=1 \mathrm{~ms}$

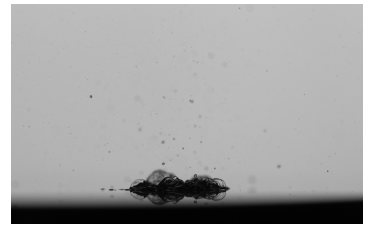

(b) $t=81 \mathrm{~ms}$

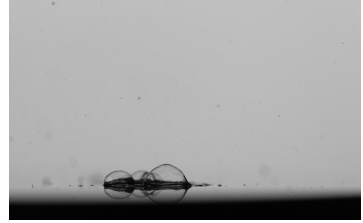

(c) $t=324 \mathrm{~ms}$

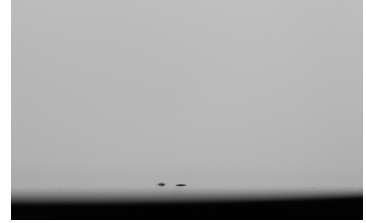

(d) $t=924 \mathrm{~ms}$

Figure 4. Water - Different phenomena for different time instants: drop impact with initial substrate temperature $T_{\mathrm{w} 0}=150^{\circ} \mathrm{C}$, drop diameter $d_{0}=2.2 \mathrm{~mm}$ and impact velocity $U_{0}=1.4 \mathrm{~m} / \mathrm{s}$.

In Fig. 3 an example of a drop impact of dilution $1: 8$ onto a hot substrate with $T_{\mathrm{w} 0}=150^{\circ} \mathrm{C}$ is shown. In the phase of drop deposition, the impact outcome is governed solely by the ratio between inertial forces that favour the spread of the drop, and the surface tension forces that govern the drop receding. Once the fluid temperature reaches the saturation point, evaporation takes place with bubbly structures appearing. Ultimately, frame (d) in both figures represents the instant of total evaporation of liquid content, with a solid residual remaining on the target surface. Compared to the water impact presented in Fig. 4, the appearance of secondary droplets in the nucleate boiling regime is not pronounced for any of the dilution ratios.

\section{Residence time}

Next, the drop residence time as a function of surface overheat was measured for three different mixture ratios. In order to obtain a comparative analysis, the experiments were supplemented with pure water batches. With a decrease in lubricant content, it is expected that the impact outcome as well as the residence time will gradually approach that of pure water.

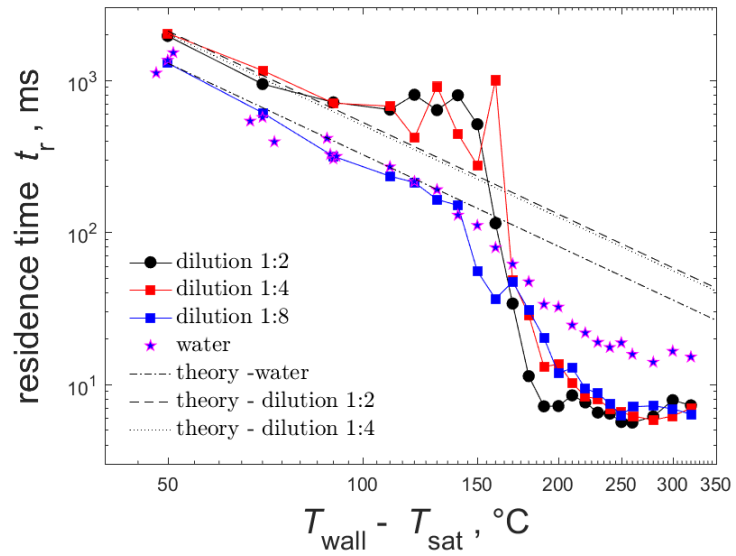

Figure 5. The residence time of the impacting drop as a function of the substrate overheat temperature for various lubricant concentrations in comparison with the theoretical predictions (6). 
Fig. 5 depicts changes in residence time with an increase in surface temperature for mixture ratios of 1:2,1:4,1:8 as well as for pure water. In Fig. 5 theoretical predictions for the residence time in the nucleate boiling regime, as given by Eq. (6), are shown.

The theoretical model for the residence time in the nucleate boiling regime for pure water provides a very accurate fit up to an overheat of $140^{\circ} \mathrm{C}$, where it can be assumed that the nucleate boiling regime terminates. Past this temperature, the regime of transition boiling occurs and the slope of the curve increases due to the increase in heat flux. Finally, the regime of film boiling sets in at an overheat temperature of $230-250{ }^{\circ} \mathrm{C}$, where the Leidenfrost point has been reached and further overheat no longer produces notable changes in the outcome. The increased concentration of lubricants is positively correlated with an increase in residence time, whereas the dilutions 1:2 and 1:4 exhibit minor differences in results.

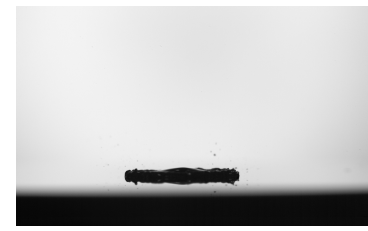

(a) $t=1.5 \mathrm{~ms}$

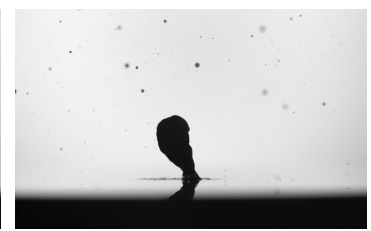

(b) $t=16.5 \mathrm{~ms}$

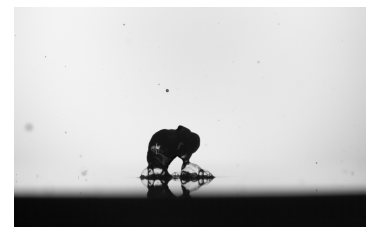

(c) $t=241 \mathrm{~ms}$

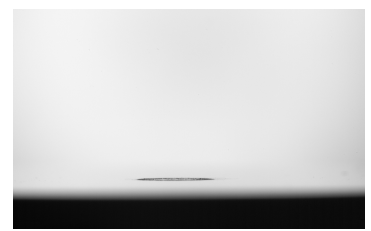

(d) $t=755.2 \mathrm{~ms}$

Figure 6. "Carpet boiling" - Different phenomena for different time instants: drop impact with initial substrate temperature $T_{\mathrm{w} 0}=220^{\circ} \mathrm{C}$, drop diameter $d_{0}=2.3 \mathrm{~mm}$ and impact velocity $U_{0}=1.7 \mathrm{~m} / \mathrm{s}$.

By increasing the temperature, both the $1: 2$ and 1:4 curves reach a plateau in the range of $100-150^{\circ} \mathrm{C}$ overheat, where the scattering of data becomes notable, thus identifying the transition boiling regime. In this regime, the residence time of the mixture drops is much longer in comparison with pure water drops. This effect can be explained in Fig. 6. The drop boiling is significantly influenced by a layer of deposited solid particles. These particles introduce the thermal resistance in the system, influencing the heat transfer between the wall and the drop. Moreover, the layer of the particles is less wettable than the polished stainless steel surface. Correspondingly the wetted area is smaller and thus the residence time becomes larger. This new regime, typical only for the mixture drops, will be called the "boiling on a carpet", or "carpet boiling", referring to the layer of deposited particles as a thermal conductivity carpet.

Above a temperature of $200^{\circ} \mathrm{C}$ overheat, abrupt termination of the transitional boiling regime occurs and all curves fall to a lower plateau. For pure water drops in this temperature range, different outcomes can be observed, such as: drop impact, drop spreading, drop receding and drop rebound. In contrast, for the mixture drops, no receding can be observed. After impact, mixture drops spread, then levitate and finally, thermal rebound can be observed. This behaviour is in accordance with lower residence times of the mixture drops in this temperature range. Interestingly, the range of film boiling appears to be independent of the lubricant concentration (in contrast to the nucleate boiling regime). This indicates that the residence time at high temperatures is not determined by the drop evaporation, but by the drop rebound time, which is comparable with the characteristic time of the drop capillary oscillations [14].

Since a very good prediction of residence time in the nucleate boiling regime has been achieved with the theoretical model for water, a least squares fit of the theoretical model with the dilutions $1: 2$ and 1:4 was performed, which is presented in Fig. 5. As can be seen, the model identifies the end of the nucleate boiling regime for both dilutions at around $90^{\circ} \mathrm{C}$ overheat. This indicates that the addition of lubricant significantly reduces the threshold temperature that separates the nucleate boiling and film boiling regimes. Previous analysis indicated that the addition of a lubricant affects the residence time in a nonlinear fashion. For dilutions $1: 2$ and 1:4, it appears that the change in concentration exhibits little or no effect on residence time, whereas for the dilution 1:8, the difference is crucial, albeit as expected, close to pure water. 


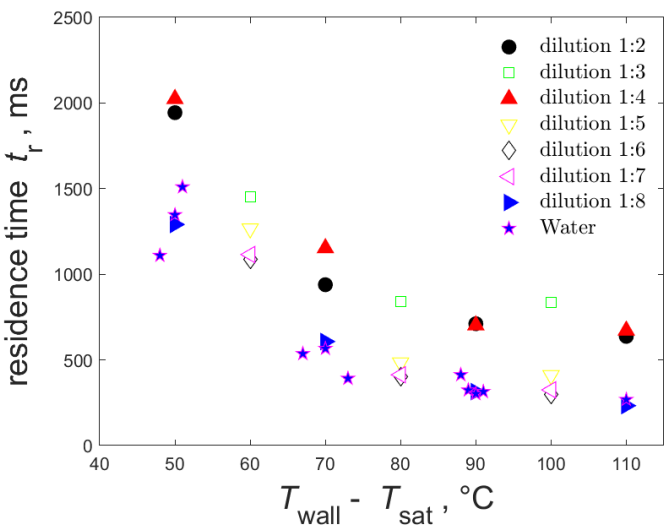

(a) Residence time of mixtures in concentrations 1:2 to $1: 8$.

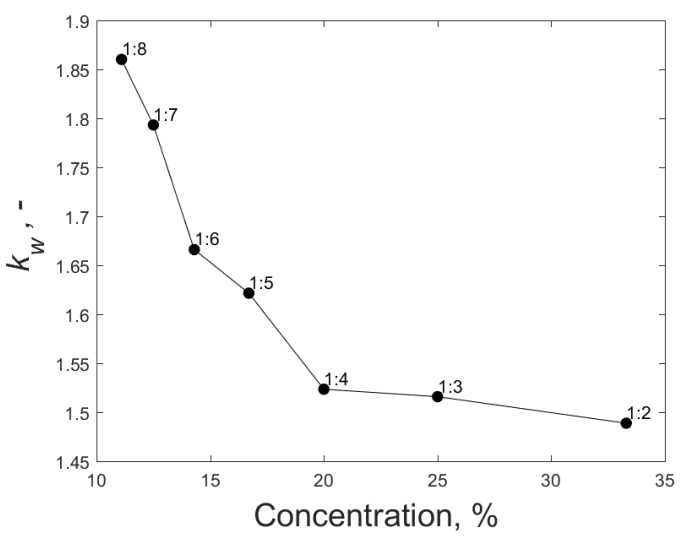

(b) Dependence of the empirical constant $k_{\mathrm{w}}$, defined in Eq. (6), on the lubricant concentration.

Figure 7. Residence time of the lubricant drop in the nucleate boiling regime.

In order to further evaluate the impact of lubricant addition, additional experiments in the nucleate boiling regime for four additional dilution ratios (1:3,1:5,1:6 and 1:7) were performed. Changes in residence time in dependence of overheat temperature for the full set of mixtures is presented in Fig. 7(a). Again a least squares regression of the model for each dilution was performed and the results are presented in Fig. $7(\mathrm{~b})$ for the parameter $k_{\mathrm{w}}$. As can be seen, for very high concentrations of lubricants (1:4 and higher), the residence time is practically completely governed by the lubricant properties, and the parameter $k_{\mathrm{w}}$ reaches an asymptotic value. For very low concentrations of lubricants $(1: 8)$ and lower, the process is governed mainly by the water (recall that the optimal value of $k_{\mathrm{w}}$ for water is 1.9 ).

\section{Conclusions}

Outcomes of a single, lubricant containing drop impact onto a heated surface have been experimentally investigated. The temperature of the impact surface has been varied in a range corresponding to the nucleate boiling, transition boiling and film boiling regimes. In order to evaluate an industrially relevant situation, the composition of the drop was varied by adding of graphite based lubricants in ratios between 1:2 to 1:8. In this way, impact outcomes including dilutions of different concentration, can be compared to results using pure water. As a result of the experiments, the contact residence time in various regimes and droplet compositions has being calculated.

It was found that the resulting properties of the drop impact vary non-linearly with an increase of lubricant concentration. For lower surface temperatures, i.e., in the nucleate boiling regime, the residence time increases by almost a factor of two after lubricant addition. Lubricant addition also affects the onset of the transition boiling regime towards the lower temperatures. The film boiling regime is almost completely independent of the lubricant concentration.

An existing theoretical model for predicting the residence time in the nucleate boiling regime of the pure water drops has been re-calibrated in order to match the measured residence time dilutions with different concentrations. It was found that for increased lubricant concentrations, $1: 4$ and beyond, the contact residence time exhibits very little change with temperature in the nucleate boiling regime. In contrast, concentrations smaller than $1: 8$ asymptotically tend to the pure water outcome.

\section{Acknowledgements}

The authors gratefully acknowledge financial support from the Deutsche Forschungsgemeinschaft (DFG) in the framework of SFB-TRR 75 (TP T02, project number 84292822). 


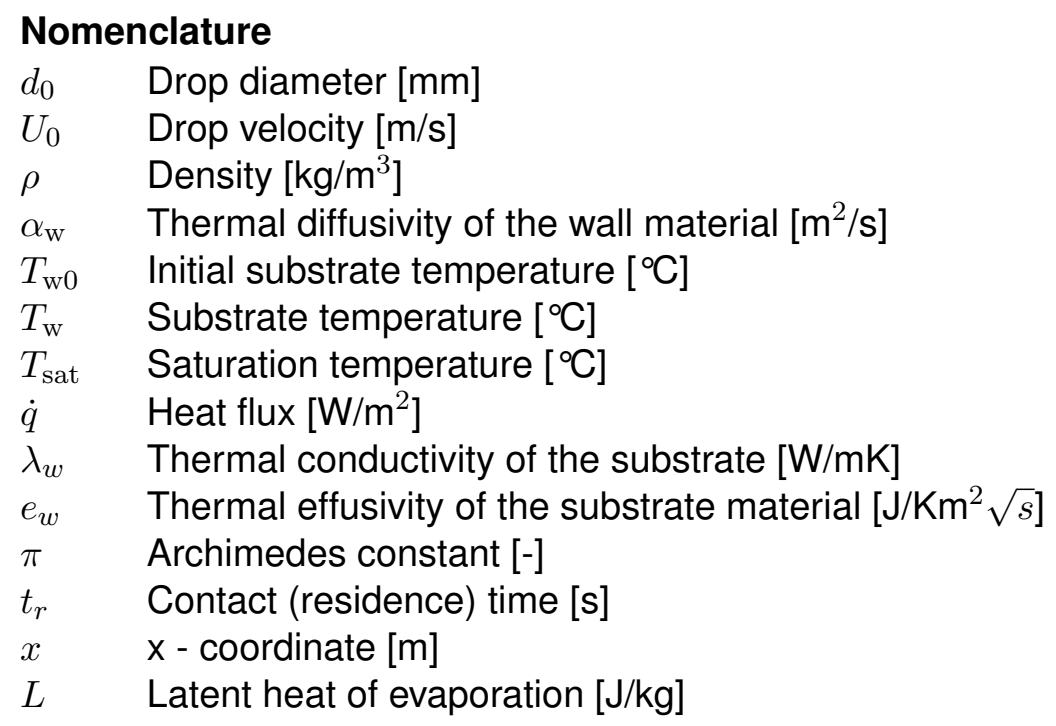

\section{References}

[1] Chen, S.-J., Tseng, A. A., 1992, International Journal of Heat and Fluid Flow, 13 (4) pp. 358-369.

[2] Cheng, W.-L., Zhang, W.-W., Chen, H., Hu, L., 2016, Renewable and Sustainable Energy Reviews, 55 pp. 614-628.

[3] Lefebvre, A., Mcdonell, V., 2017, Atomization and Sprays, Second Edition, CRC press.

[4] Liang, G., Mudawar, I., 2017, International Journal of Heat and Mass Transfer, 115 pp. 1174-1205.

[5] Liang, G., Mudawar, I., 2017, International Journal of Heat and Mass Transfer, 115 pp. 1206-1222.

[6] Tenzer, F., Roisman, I. V., Tropea, C., 2019, Journal of Fluid Mechanics, 881 pp. 84-103.

[7] Breitenbach, J., 2019, Drop and spray impact onto a hot substrate: Dynamics and heat transfer, Ph.D. thesis, Technische Universität Darmstadt, Darmstadt, Germany.

[8] Breitenbach, J., Roisman, I. V., Tropea, C., 2018, Experiments in Fluids, 59 pp. 1-21.

[9] Yarin, A. L., Roisman, I. V., Tropea, C., 2017, Collision phenomena in liquids and solids, Cambridge University Press.

[10] Yarin, A. L., 2005, Annual Review of Fluid Mechanics, 38 pp. 159-192.

[11] Batzdorf, S., Fischer, S., Gambaryan-Roisman, T., Stephan, P., 2013, International Journal of Heat and Mass Transfer, 61 pp. 605-614.

[12] Rioboo, R., Marengo, M., Tropea, C., 2001, Atomization and Sprays, 11 pp. 155-166.

[13] Marengo, M., Antonini, C., Roisman, I. V., Tropea, C., 2011, Current Opinion in Colloid \& Interface Science, 16 pp. 292-302.

[14] Schmidt, J. B., Hofmann, J., Tenzer, F., Breitenbach, J., Tropea, C., Roisman, I. V., 2020, Thermosuperrepellency of a hot substrate caused by vapour percolation, Preprint available at Research Square, https://doi.org/10.21203/rs.3.rs-56255/v.

[15] Lubritech, F., 2021, Lubrodal F105, https://www.fuchs.com/lubritech/en/product/ product/118075-lubrodal-f-105/.

[16] Roisman, I., 2010, Journal of Fluid Mechanics, 656 pp. 189-204.

[17] Breitenbach, J., Roisman, I. V., Tropea, C., 2017, Physical Review Fluids, 2 p. 074301. 